\title{
Photoproduction of Pseudoscalar Mesons
}

\author{
R. A. Arndt ${ }^{\mathrm{a}}$, W. J. Briscoe ${ }^{\mathrm{a}}$, G. V. O’Rielly ${ }^{\mathrm{b}}$, I. I. Strakovsky ${ }^{\mathrm{a}}$, R. L. Workman ${ }^{\mathrm{a}}$ \\ aThe Center for Nuclear Studies, Department of Physics, \\ The George Washington University, Washington, D.C. 20052 \\ bepartment of Physics, University of Massachusetts Dartmouth, M.A. 02747
}

Experiments that study the photoproduction of pseudoscalar mesons; pions, etas and kaons, have the potential to increase our knowledge of baryon and hyperon resonance properties. Recent experiments at JLab, Mainz, GRAAL, and Bonn are beginning to produce results in the form of polarization and asymmetry measurements and determinations of the differential and integrated cross sections. These new data are essential to the performance of Partial-Wave Analyses that are less model dependent and coupledchannels calculations that incorporate unitarity dynamically, combining hadronic reaction channels together with electromagnetic processes. This approach is necessary to extract resonance properties and may lead to the identification of missing, but predicted, resonances. Some recent experimental and phenomenological results for single and double pseudoscalar meson photoproduction are discussed.

Studies of the baryon spectrum have generally been data-base driven. While most states were first detected through hadronic scattering (mainly pion-nucleon), present research is focused on resonance excitation by real and virtual photon probes. The photo- and electroproduction of pseudoscalar mesons has been studied most intensively for a number of practical reason. First, in the case of pion production, there is a clear and direct connection to the extensive results from elastic pion-nucleon scattering. Here one benefits from knowing, in advance, the resonances which couple most strongly to the final state. Second, in general, pseudoscalar-meson production is relatively easy to analyze. Many additional amplitudes contribute to the production of vector mesons, for example. However, if one is looking for "missing resonances," states not found in the elastic scattering or electromagnetic production of single pseudoscalar mesons, the search must be extended to processes having other final states (such as two-meson productions).

It has become clear over the last decade that any attempt at an unambiguous description of resonances requires a comprehensive approach to the analysis of all contributing reactions. In order to be able to perform such coupled-channel analyses, one needs easy access to all related sets of experimental data, in addition to a theoretical and calculational framework for a common description. The availability of partial-wave amplitudes greatly simplifies numerical aspects of coupled-channel analysis. The number of fitted partial-wave amplitudes associated with a dataset may be smaller than the count of individual data by orders of magnitude, and can account for issues associated with statistical 
and systematic errors and the rejection of inconsistent measurements.

In Fig. 1, we compare the SAID and MAID fits to existing data and preliminary CLAS data for single-pion photoproduction. Note that the variation in fits to existing data would be unacceptable if only CLAS data were retained. Differences, usually minor in the unpolarized cross sections, are magnified in predictions of single and double polarization observables.

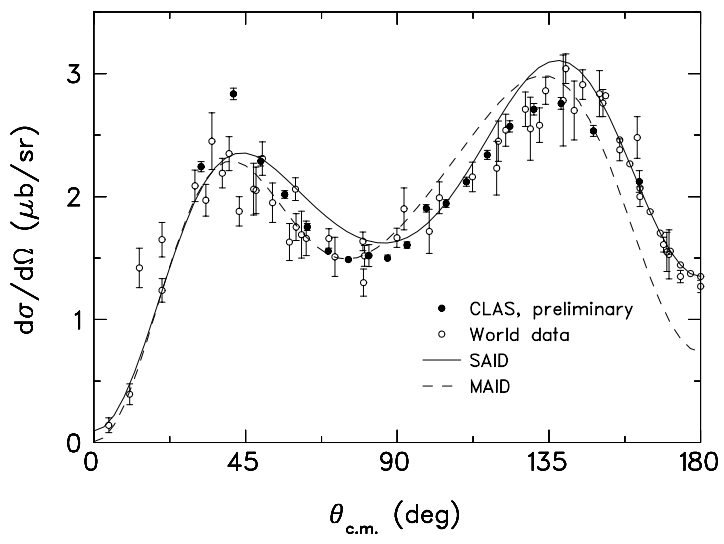

Figure 1. Preliminary differential cross section data in the $\mathrm{p}(\gamma, \mathrm{p}) \pi^{0}$ reaction at $\mathrm{E}_{\gamma}$ $=1000 \mathrm{MeV}$ from the CLAS $g 1 c$ run group (solid circles) [1]. Data are compared to the world data (open circles), along with SM02 [ 2] solution (solid line) and MAID [3] (dashed line) predictions.

In Fig. 2, we compare SAID and MAID results for the differential and total cross section (polarized beam-target) contributing to the the GDH sum rule. The inclusion of this and other precise polarization data has led a number of groups to propose changes to supposedly well-known resonance states. Note that the single-pion contribution to the sum rule is negligible by $2 \mathrm{GeV}$.
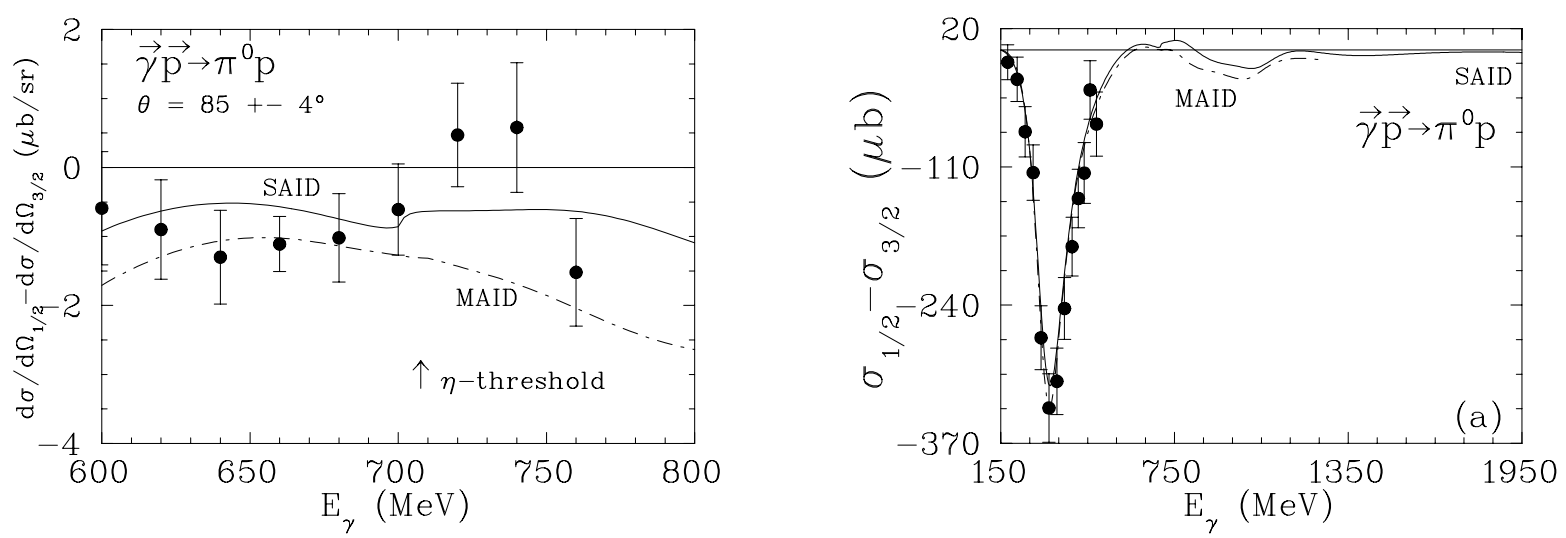

Figure 2. Difference of the (a) differential and (b) total cross sections for the helicity states $1 / 2$ and $3 / 2$ for $\vec{\gamma} \vec{p} \rightarrow \pi^{0} p$. The solid (dash-dotted) line represents the SM02 [ 2] (MAID2001 [ 3]) solution. Experimental data are from Mainz [ 4, 5].

In Fig. 3, we show results for double-pion photoproduction, which displays structures 
which have been attributed to states not strongly seen in single-pion production. Here, however, interpretation is more difficult, again motivating a multi-channel treatment.

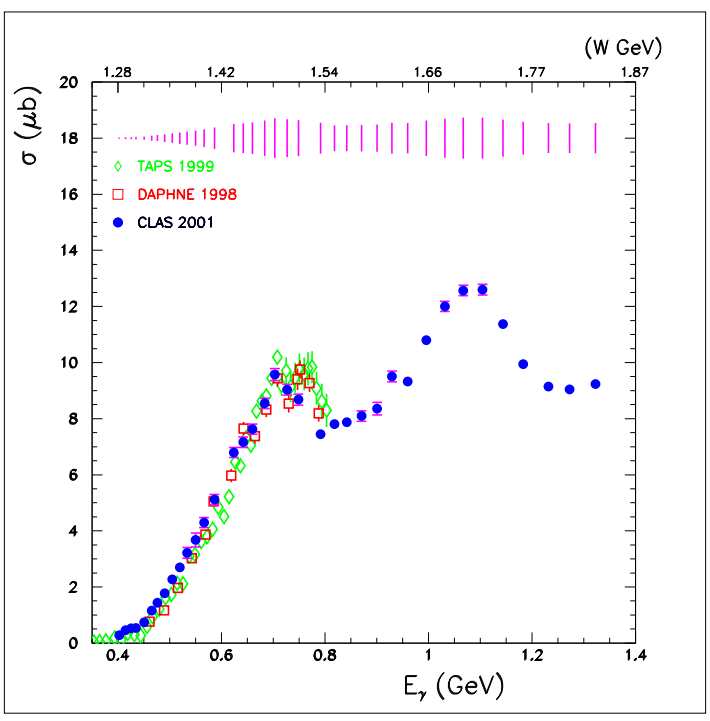

Figure 3. Total cross section for the $\gamma p \rightarrow p \pi^{0} \pi^{0}$ reaction. Preliminary CLAS data [6] are compared to the world data (open circles) obtained with the DAPHNE'98 and TAPS'99 detectors. CLAS data are plotted with statistical errors. The band at the top of the figure delineates the range of systematic uncertainties.

In Fig. 4, two GW fits to the S-wave dominated eta-photoproduction differential cross sections are displayed. This reaction has the potential to give cleaner information on the $\mathrm{N}(1535)$ resonance, the pion channel being masked by a sharp cusp effect (due to the etanucleon threshold). Note that both fits, and also a fit from the Mainz group, tends to drop off rapidly at very forward angles. An interesting preliminary set of data from GRAAL suggests a flatter extrapolation to the forward direction, which would necessitate a reexamination of the underlying assumptions in most fits (a smoother behaviour appears to be predicted by the model of Li and Saghai.)

Fits to pion electroproduction and kaon photoproduction have also been completed, and a multi-channel K-matrix approach to eta photoproduction is being explored. Further fits and an expansion of our on-line database is expected in the near future.

\section{Acknowledgments}

This work has been supported by U.S. Department of Energy grant DE-FG02-99ER41110. Additional support has been provided by Jefferson Lab and the Southeastern Universities Research Association through the D.O.E. contract DE-AC05-84ER40150. 


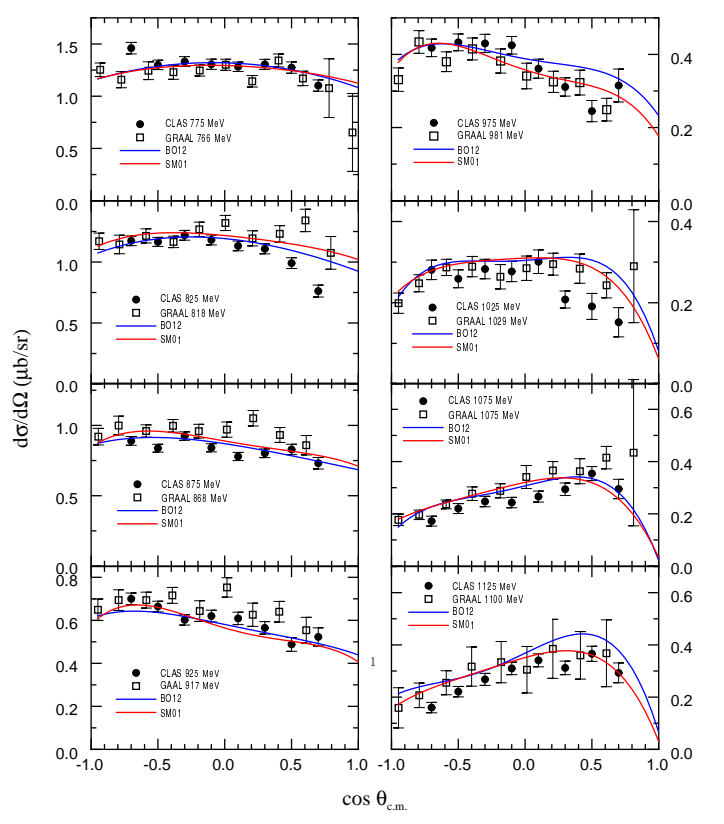

\section{REFERENCES}

1. M. Wood, private communication, 2002.

2. R. A. Arndt, W. J. Briscoe, I. I. Strakovsky, and R. L. Workman, to be published in Phys. Rev. C 66 (2002) 0552xx; http://gwdac.phys.gwu.edu

3. Mainz fits are available at the MAID website http://www.kph.uni-mainz.de/MAID/. See also S. S. Kamalov et al., Phys. Rev. C 64 (2001) 032201. The Unitary Isobar Model is developed at Mainz (hereafter called MAID), D. Drechsel et al., Nucl. Phys. A645 (1999) 145. MAID2000 refers to the Feb. 2001 version of the MAID solution, MAID2001 refers to the Nov. 2001 version of the MAID solution from S. Kamalov.

4. J. Ahrens et al., Phys. Rev. Lett. 88 (2002) 232002.

5. J. Ahrens et al., Phys. Rev. Lett. 84 (2000) 5950.

6. S. Philips, Ph.D. Thesis, The George Washington University, Jan. 2002.

7. M. Dugger et al., Phys. Rev. Lett. 89 (2002) 222002.

8. F. Renard et al., Phys. Lett. B528, 215 (2002).

9. R. A. Arndt, I. I. Strakovsky, and R. L. Workman, in progress; http://gwdac.phys.gwu.edu. 